\title{
Finite element analysis and experimental study of plastic lattice structures manufactured by selective laser sintering
}

\author{
Jie Niu ${ }^{1}$, $\underline{\text { Hui Leng Choo }}^{1}$, Wei Sun ${ }^{2}$ \\ ${ }^{1}$ The University of Nottingham Ningbo China, 199 Taikang East Road, Ningbo, China. \\ ${ }^{2}$ The University of Nottingham, Nottingham, NG7 2RD, UK.
}

\begin{abstract}
The availability of Additive Manufacturing (AM) technologies in particular the selective laser sintering (SLS) process has enabled the fabrication of high strength, lightweight and complex cellular lattice structures. In this study, the effective mechanical properties of SLS produced periodic lattice structures were investigated. Three different types of lattice structures were designed by repeating three types of open-form unit cells consisting of triangular prism, square prism, and hexagonal prism. A novel approach of creating the complex and conformable lattice structures using traditional modelling software such as Creo ${ }^{\circledR}$ proposed by the authors was used. Based on the predesigned lattice structures, finite element analysis (FEA) was carried out to evaluate the mechanical properties of these structures. For the experimental study, nylon samples were printed using a plastic selective laser sintering system and tested using a universal testing machine. FEA results show that lattice structures with triangular prism perform better than the other two prisms in terms of Young's modulus to relative density ratio. Tensile tests results show good conformance with the results obtained from FEA.
\end{abstract}

Keywords: lattice structure, unit cell, additive manufacturing, selective laser sintering, properties

Corresponding author: Hui Leng Choo, The University of Nottingham Ningbo China, 199 Taikang East Road, Ningbo 315100, China. Email: huileng.choo@ nottingham.edu.cn 


\section{Introduction}

Modern materials and lightweight design concepts such as composites, cellular materials or sandwich structures and topology design have a high potential of improving the performance of a product. Recently, additive manufacturing (AM) has received considerable attention in lightweight design in automotive, aerospace, and biomedical applications [1]. For a given design block with some certain materials, topology optimization approach based on maximum stiffness design criteria has no adequate capabilities for hierarchical design integrations at multiple scales [2]. On the contrary, lattice structure composed by uniform unit cells is another approach attempting to achieve the balance between design efficiency and design accuracy, as well as tailored mechanical properties [3]. As a new solution in lightweight green manufacturing, lattice structure addresses well for complex full-freedom design and optimization where other traditional manufacturing technologies become largely impractical [4-6]. By far, various unit cells have been studied on the basis of selective laser melting (SLM) [7-9] and selective laser sintering (SLS) [10-12]. Hao et al. [2] presented an approach to generate and design periodic lattice structures and investigated the manufacturability of some selected structures using SLM process. In their work, two unit cell types, the Schoen Gyroid and Schwartz Diamond were considered. The results revealed that the Schoen Gyroid and Schwartz Diamond cellular structures with the same volume fraction of $15 \%$ and different unit cell sizes of $2 \mathrm{~mm}, 5 \mathrm{~mm}$ and $8 \mathrm{~mm}$ were manufacturable through SLM. Yang et al. [2] also explored a unit-cell-based modelling approach that integrated experimentation and limited-scale simulation for lightweight cellular lattice structure. This approach could potentially lead to computationally efficient design optimizations with the lightweight structures by SLM. While in SLS areas, plastic materials were commonly used. For example, Nylon 6 material was used to fabricate reverse engineered 
structures for use in the optimization and simulation processes of the additively manufactured structures [13]. Gandhi et al. investigated the most effective lattice cell layout, the design, modelling, production and validation of a compression block with Octet Framed-based unit cells [13]. Maskery et al. [12] studied the deformation processes and mechanical performance of several functionally graded and non-graded lattice structures using body-centred-cubic (BCC) and a reinforced variant called BCCz by SLS. They found that BCCz lattices possess remarkable mechanical anisotropy, being weaker when their reinforcing struts were perpendicularly applied, while BCC lattices tended to be more isotropic. Kinstlinger et al.[11] concluded for structures produced by SLS that mechanical properties vary between geometries and may be markedly different from the bulk material properties.

An important challenge in realizing engineered lattice structures is the variation in detailed shapes and sizes of the struts that compose the lattice due to the resolution limitations of AM process. Many researches have focused on the evaluation of mechanical properties of lattice structures over the past decades. Mathematical relationships or theoretical models [14-17], experimental study [2, 3, 9, 18] and Finite Element Method (FEM) [7, 13, 19, 20], have been proposed to estimate the mechanical properties such as Young's modulus and ultimate strength. In the work done by Park et al. [21], they investigated the elastic modulus of lattice structure filled with square unit cells. The results showed that the elastic modulus varied with strut diameters and rotation angles. Papers in this area only focus on one specific unit cell and describe its mechanical properties by simple and limited number of samples. As pointed out by Jin et al. [22], an overall understanding of specimen size effects on the mechanical properties (modulus, strength, plateau stress and densification strain) in out-of-plane and in-plane directions is still unclear. 
In this paper, the mechanical properties of lattice structures formed by three types of unit cells were investigated. The effects of the different types of unit cells and unit cell dimensions on mechanical properties were studied via finite element analysis (FEA). Tensile tests were carried out using nylon samples manufactured by SLS process to verify the FEA results.

\section{Methodology}

Geometry modelling

(a)
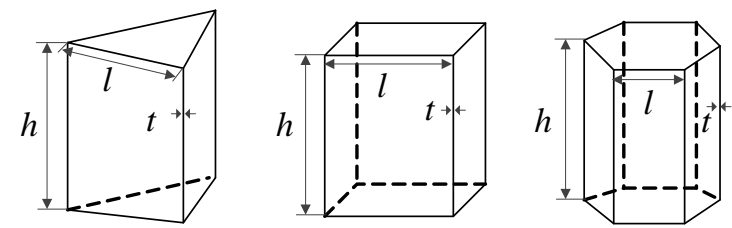

(b)
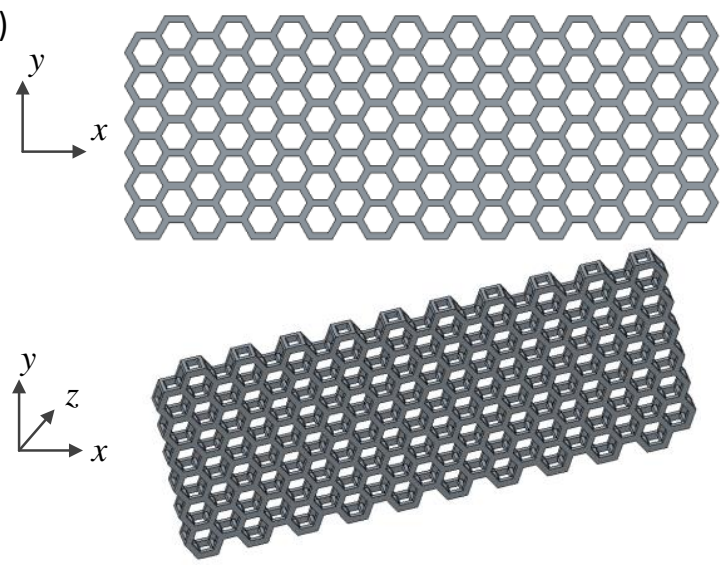

(c)
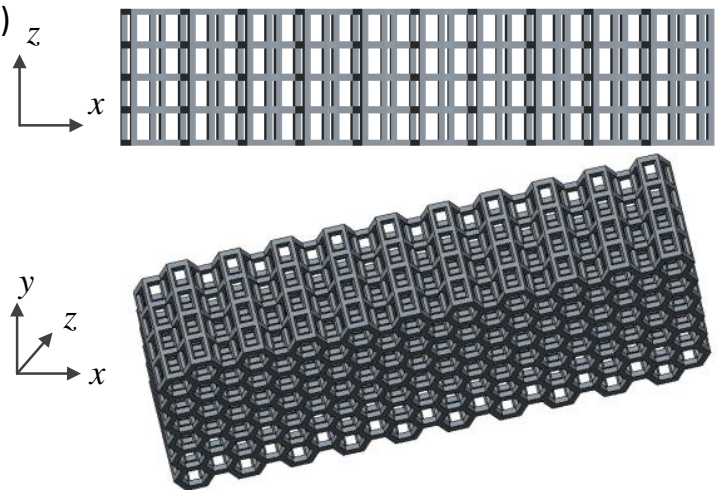

Figure 1 Geometrical evolution of lattice structures with three unit cells, (a) 3D skeleton unit cells of triangular prism, square prism and hexagonal prism, (b) patterning method in in-plane, (c) patterning method in out-plane. 
Creo® was used to create the lattice structures used in FEA and for manufacturing using SLS process. As shown in Figure 1 (a), three regular unit cells of triangular prism, square prism and hexagonal prism are defined by length, $l$, strut thickness with square cross-section, $t$, and height, $h$. To create the lattice structure with large scale unit cells, one single unit cell is firstly patterned in the in-plane direction (Figure 1 (b)), namely $\mathrm{x}$ - and y-direction with patterning distance determined by $l$. This single layer is then repeated in the out-plane direction (z-direction), where the distance is equal to $h$ between neighbouring layers as displayed in Figure 1 (c). Finally, the original lattice structure is trimmed by a given block with dimensions of $20 \times 20 \times 50 \mathrm{~mm}$ with all geometry symmetry in each direction as shown in Figure 2 (a) - (c). The tailored lattice structure has irregular boundaries which would result in incomplete unit cells which do not contribute to the structure, as pointed out by Li [4], or cause stress free boundary as illustrated by Onck et al. [22]. This should be avoided because it is inconsistent with the stress condition at the boundary of the original solid part and the irregular cutting edges are usually non-convergent in FEA. The solution in this project is that the irregular boundaries are connected and covered by ribs and caps as shown in Figure 2 (d). The cross-section of the ribs used is equal to that of the strut and the thickness of the caps is $1 \mathrm{~mm}$. With these design constraints, it intends to make it comparable that the only variation for the lattice structure is the shape parameters of the unit cell. Table 1 shows the geometry parameters of the unit cells investigated in this project. These parameters were determined based on the resolution capability of the SLS process. 
(a)

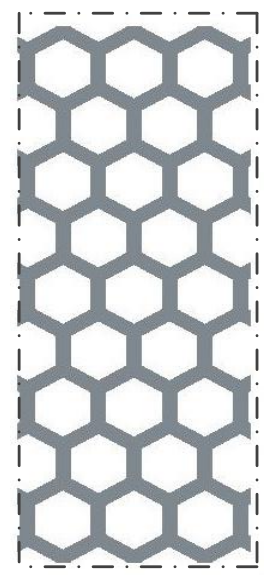

(b)

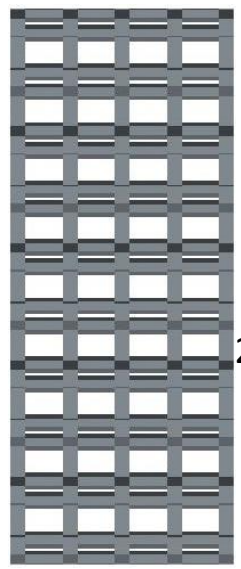

(c)

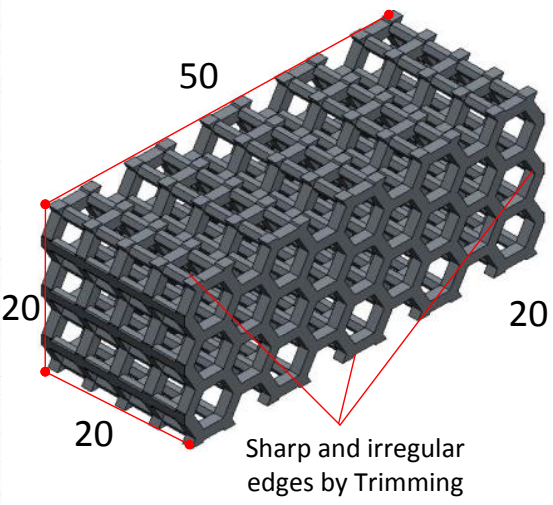

(d)

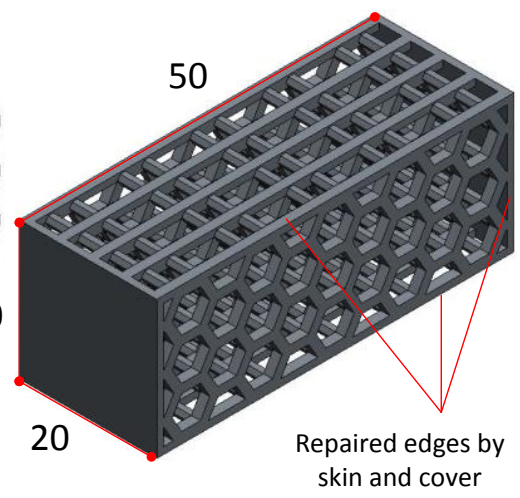

Figure 2 Skimming for uniformly distributed unit cells and boundary treatment by Creo®, (a) profile of tailored structure in inplane direction, (b) profile of tailored structure in out-plane direction, (c) trimmed lattice structure with initial general dimension, (d) post-processed conformal structure with ribs and caps. (Units: $\mathrm{mm}$ )

Table 1 Geometry parameters of the unit cells used to build the models investigated in this project.

\begin{tabular}{ccccc}
\hline \multirow{2}{*}{ Sample } & \multirow{2}{*}{ Shape of unit cell } & \multicolumn{3}{c}{ Geometry parameters of unit cells } \\
\cline { 3 - 5 } & & $l / \mathrm{mm}$ & $t / \mathrm{mm}$ & $h / \mathrm{mm}$ \\
\hline 1 & Cubic & 3.20 & 1.40 & 4.65 \\
2 & Cubic & 4.50 & 1.00 & 4.75 \\
3 & Hexagonal & 2.20 & 1.40 & 4.65 \\
4 & Hexagonal & 2.80 & 1.00 & 4.75 \\
5 & Triangular & 4.80 & 0.92 & 4.77 \\
6 & Triangular & 4.80 & 1.04 & 4.74 \\
\hline
\end{tabular}

\section{Material parameters}

The material used in this project was Nylon 12 (PA2200 from EOS GmbH). Young's modulus and tensile strength of selective laser sintered Nylon 12 are comparable with values for standard injection moulded samples, but the ductility is at least an order of magnitude lower [23]. The published mechanical properties of PA2200 are listed in Table 2. The Young's modulus and Poisson's ratio used in this project for FEA are $1700 \mathrm{MPa}$ [23] and 0.33 respectively. 
Table 2 Mechanical properties of SLS Nylon 12 (PA2200, EOS GmbH) [23].

\begin{tabular}{|c|c|c|c|c|c|}
\hline $\begin{array}{c}\text { Tensile strength } \\
(\mathrm{MPa})\end{array}$ & $\begin{array}{c}\text { Tensile modulus } \\
(\mathrm{MPa})\end{array}$ & $\begin{array}{c}\text { Tensile elongation at } \\
\text { break }(\%)\end{array}$ & $\begin{array}{c}\text { Part melting } \\
\left({ }^{\circ} \mathrm{C}\right)\end{array}$ & $\begin{array}{l}\text { Particle size, } \\
\text { average }(\mu \mathrm{m})\end{array}$ & $\begin{array}{c}\text { Part moisture absorption, } \\
23{ }^{\circ} \mathrm{C}(\%)\end{array}$ \\
\hline 45 & 1700 & 20 & 184 & 58 & 0.52 \\
\hline
\end{tabular}

\section{Finite element analysis}

Lightweight structures pose a special challenge to FEA. In addition to the global response of a structure, careful analysis of local details such as areas of load introduction or localized instabilities can be crucial for reliable predictions [2]. In this work, the combination of quadratic tetrahedron element (tet 10) and hexahedron element (hex 20) was used. The conformable lattice structure was firstly divided into small sample parts and meshed by different element types, then emerged into a single part. Mesh-sensitivity was carried out using node number and maximum stress to select the appropriate element size. The strength converged when the element size is a quarter of the thickness of the strut. In this project, the average element quality is more than 0.95 and the skewness is lower than 0.3, indicating satisfied mesh quality [24].

An applied force $F$ was varied at $50 \mathrm{~N}, 100 \mathrm{~N}$ and $200 \mathrm{~N}$, and the corresponding deformation was obtained by FE models. To calculate the effective Young's modulus of each nylon sample, a gauge length of $25 \mathrm{~mm}$ within the central area is selected. As shown in Figure 3, AA' and BB' are the left and right side of the effective part, where $D_{L}$ is the deformation at left side and $D_{R}$ indicates the deformation along the right side. The values of $D_{L}$ and $D_{R}$ can be extracted in ANSYS by the marked planes. 


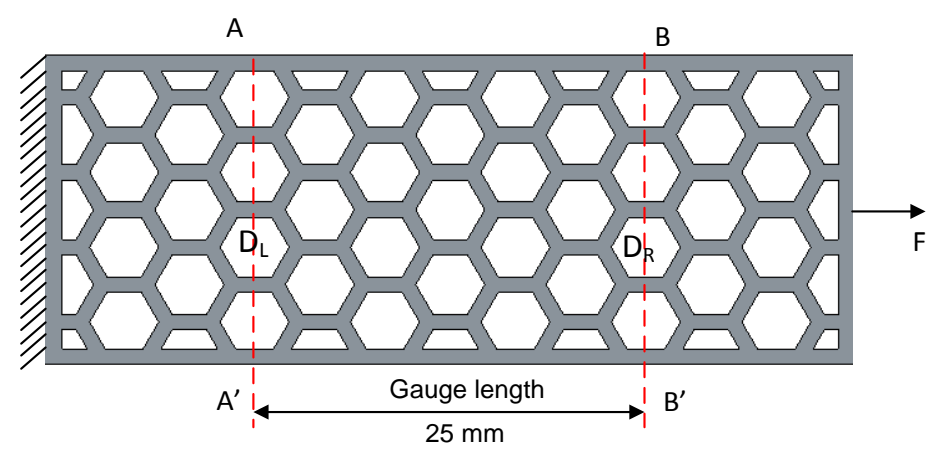

Figure 3 Gauge length determined in the workpiece.

\section{Experimental sample preparation}

Samples listed in Table 1 were fabricated using SLS system FORMIGA P110 from EOS. The material used was PA2200 as described previously. Four samples of each model were printed using the parameters listed in Table 3. All samples were placed in the $\mathrm{x}$-direction as shown in Figure 4 to obtain maximum Young's modulus.

Table 3 Sintering process parameters.

\begin{tabular}{ll}
\hline Laser power & $16 \mathrm{~W}$ \\
Laser type & $\mathrm{CO}_{2}, 30 \mathrm{~W}$ \\
Laser spot diameter & $0.40 \mathrm{~mm}$ \\
Laser scan spacing & $0.15 \mathrm{~mm}$ \\
Layer thickness & $0.10 \mathrm{~mm}$ \\
Laser scan speed & $5000 \mathrm{~mm} / \mathrm{s}$ \\
Laser scan strategy & $\mathrm{EOS} \mathrm{sorted}$ \\
Particle size & $58 \mathrm{um}$ \\
Temperature of process chamber & $168^{\circ} \mathrm{C}$ \\
Temperature of removal chamber & $157^{\circ} \mathrm{C}$ \\
Environment temperature & $23{ }^{\circ} \mathrm{C}$ \\
\hline
\end{tabular}




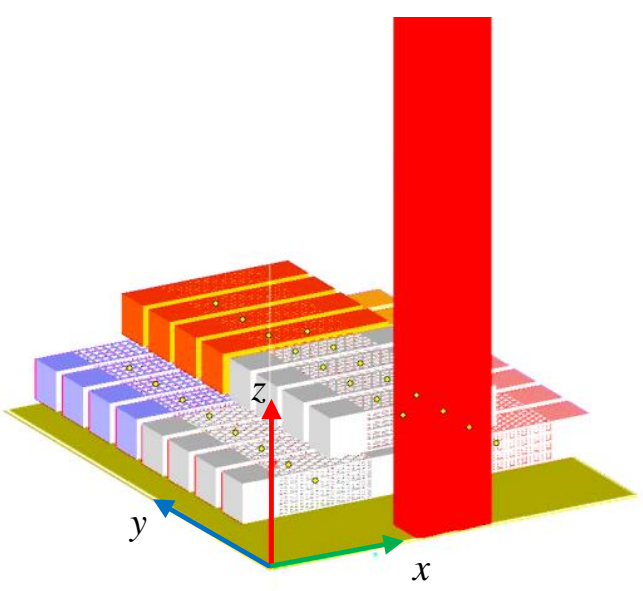

Figure 4 Position of workpieces in the platform of Magics.

\section{Tensile tests}

Tensile tests were carried out using Instron 8950 universal testing machine. Each model was tested at least three times to obtain the average Young's modulus. The tests were carried out at a speed of $2.5 \mathrm{~mm} / \mathrm{min}$. Extensometer with a gauge length of $25 \mathrm{~mm}$ was used to measure the deformation of the samples for Young's modulus calculation.

\section{Results and Discussion}

\section{FEA results}

Table 4 shows the Young's modulus results obtained from FEA ( $\left.\mathrm{E}_{\mathrm{T}}-\mathrm{FEA}\right)$, relative density (RD) and ratio of $\mathrm{E}_{\mathrm{T}}-\mathrm{FEA}$ to $\mathrm{RD}\left(\mathrm{E}_{\mathrm{RD}}\right)$. As each sample has a different $\mathrm{RD}$, which represents the amount of material available to withstand a given load, it becomes unreliable to compare the $\mathrm{E}_{\mathrm{T}}$ values directly. Hence, $\mathrm{E}_{\mathrm{RD}}$ is more appropriate for comparison. In general, increasing $\mathrm{RD}$ increases the $\mathrm{E}_{\mathrm{RD}}$ for each of the unit cell shape although at varying degrees. Comparing Sample 2, 4 and 5 with similar RD, triangular unit cell is the strongest, followed by cubic and hexagonal unit cell. This result is consistent with results for triangular honeycomb compared with the other 
two unit cells $[16,25]$. The results obtained also show the critical sensitivity of unit cell dimensions to the properties of the lattice structures. For triangular unit cell, with a small increase in $t$ of $0.12 \mathrm{~mm}$ for Sample 5 and 6 ( $l$ remains constant), RD increases 3.75 times while $\mathrm{E}_{\mathrm{T}}$ and $\mathrm{E}_{\mathrm{RD}}$ increase by around $340 \%$ and $18 \%$ respectively. However for cubic unit cell, a change in both $l$ and $t$ do not have significant effect on $\mathrm{E}_{\mathrm{RD}}$ even with a 2.5 times difference in RD. Further studies in the future will focus on investigating and quantifying the effects of each unit cell parameters on the $\mathrm{E}_{\mathrm{T}}$ of the lattice structure.

Table 4 Calculation of effective Young's modulus by linear forces and responding deformations by FEA, relative error, relative density and ratio of $\mathrm{E}_{\mathrm{T}}-\mathrm{FEA}$ to $\mathrm{RD}$.

\begin{tabular}{ccccccc}
\hline Sample & Cell shape & Ет-FEA/MPa & Ет-Exp/MPa & $\delta / \%$ & Relative density (RD) & Eт to RD (ERD)/MPa \\
\hline 1 & Cubic & 269 & $254 \pm 5.5$ & 5.8 & 0.25 & 1076 \\
2 & Cubic & 113 & $107 \pm 4.1$ & 6.0 & 0.10 & 1130 \\
3 & Hexagonal & 211 & $200 \pm 6.3$ & 5.5 & 0.28 & 753 \\
4 & Hexagonal & 48 & $45 \pm 0.2$ & 5.7 & 0.08 & 600 \\
5 & Triangular & 157 & $148 \pm 3.0$ & 5.1 & 0.12 & 1308 \\
6 & Triangular & 691 & - & - & 0.45 & 1536 \\
Solid & & - & 1700 & - & 1.00 & 1700 \\
\hline
\end{tabular}

\section{Quality of manufactured samples}

Tensile test samples produced by SLS process is shown in Figure 5 along with the microscopic images of the unit cells in both the in-plane and out-plane directions. To protect the lattice structure from being destroyed by the tensile test grips, two solid symmetric ends were added to the meshed parts as shown in Figure 5. In this case, the total length becomes $90 \mathrm{~mm}$. All the lattice structures in Table 1 were manufacturable to good quality except for Sample 6. The gaps in the in-plane direction were too small for efficient removal of the remaining powders in the sample. Therefore, Sample 6 was not used for tensile test. 


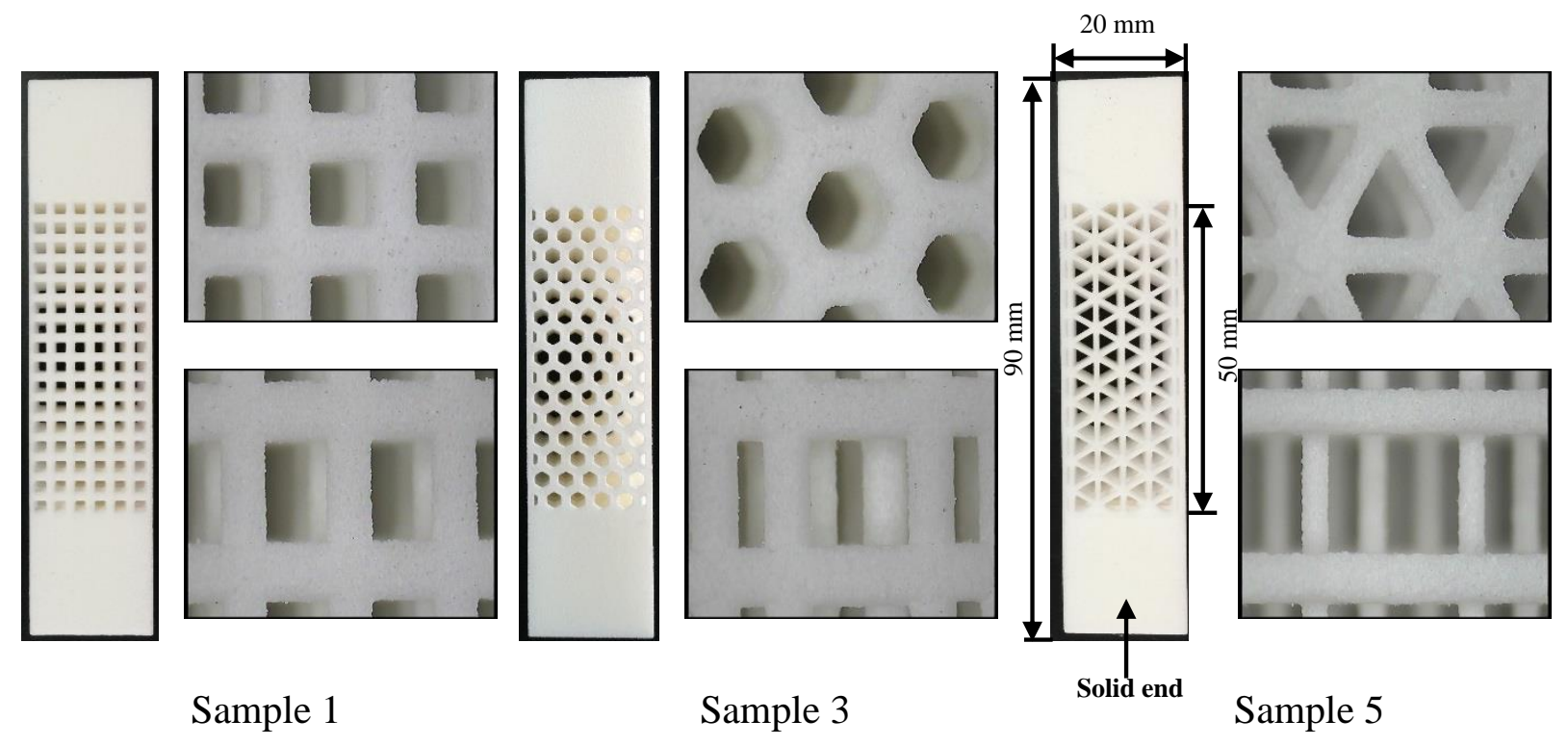

Figure 5 Samples printed by SLS process and microscopic images of the unit cell in both the in-plane and out-plane directions.

\section{Tensile tests results}

Figure 6 shows typical load-extension curve for each of the tested samples. Samples 1, 3 and 4 show classic tensile behaviour as expected with solid materials. However, Sample 5 shows several plateau of yielding beyond the maximum load. From observation during the experiment, each plateau corresponds to the complete detachment of each layer of the unit cell. Sample 2 shows similar results to Sample 5 but not as obvious. This behaviour will be further investigated in future work. 


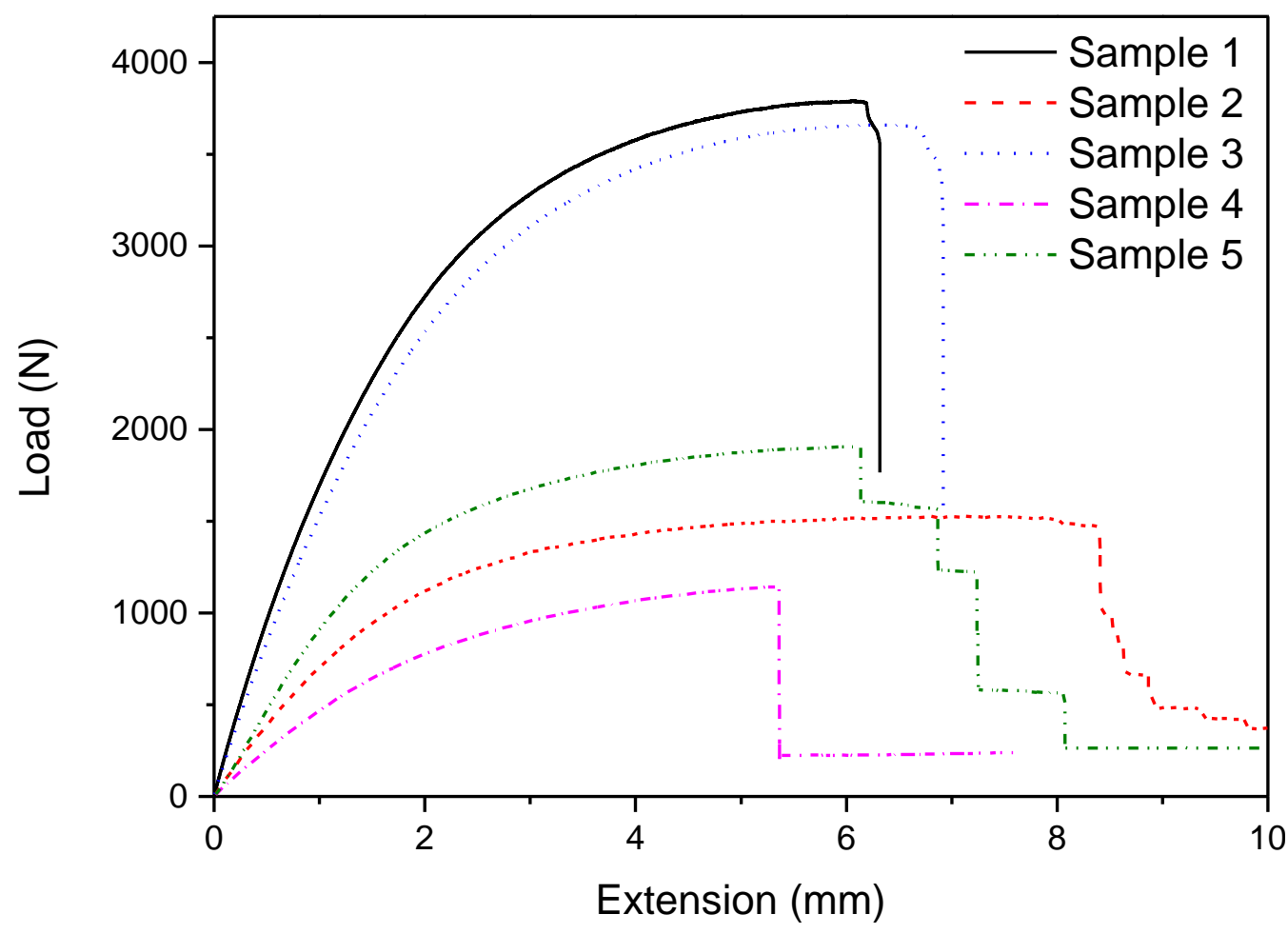

Figure 6 Load-extension curves of the five samples.

The values of Young's modulus obtained from experiments are shown in Table $4\left(\mathrm{E}_{\mathrm{T}}\right.$-Exp). Both experimental and FEA results show good conformance with a maximum error of $6.0 \%$. This shows that the FEA model in this project could be used in the future for further analysis of the effects of unit cell parameters on tensile properties of the lattice structures.

\section{Conclusions and Future Work}

FEA results showed that lattice structures with triangular prism perform better than the other two prisms in terms of tensile elastic Young's modulus to relative density ratio. Different types of unit cells and geometry parameters showed varying degrees of effects on mechanical properties of lattice structures. Young's modulus results from experiment show good conformance with the results obtained from FEA. This shows that the element types tet 10 and hex 20 are suitable to be 
used in FEA of lattice structures. SLS process was also demonstrated to be able to manufacture the samples with good surface quality and mechanical properties. In the future, the effects of geometry parameters of the unit cells will be investigated in detail. Non-linear behaviour of the plastic region and fracture of the lattice structures will also be studied in-depth.

\section{References}

1. Wong, K.V. and A. Hernandez, A review of additive manufacturing. ISRN Mechanical Engineering, 2012. 2012.

2. Hao, L. and D. Raymond. Design and additive manufacturing of cellular lattice structures. in The International Conference on Advanced Research in Virtual and Rapid Prototyping (VRAP). Taylor \& Francis Group, Leiria. 2011.

3. Ju, J. and J.D. Summers, Compliant hexagonal periodic lattice structures having both high shear strength and high shear strain. Materials \& Design, 2011. 32(2): p. 512-524.

4. Klahn, C., B. Leutenecker, and M. Meboldt, Design for Additive Manufacturing - Supporting the Substitution of Components in Series Products. Procedia CIRP, 2014. 21: p. 138-143.

5. Parthasarathy, J., B. Starly, and S. Raman, A design for the additive manufacture of functionally graded porous structures with tailored mechanical properties for biomedical applications. Journal of Manufacturing Processes, 2011. 13(2): p. 160-170.

6. Vayre, B., F. Vignat, and F. Villeneuve, Designing for Additive Manufacturing. Procedia CIRP, 2012. 3: p. 632-637.

7. Li, P., Constitutive and failure behaviour in selective laser melted stainless steel for microlattice structures. Materials Science and Engineering: A, 2015. 622: p. 114-120. 
8. Nguyen, J., S.-i. Park, and D. Rosen, Heuristic optimization method for cellular structure design of light weight components. International Journal of Precision Engineering and Manufacturing, 2013. 14(6): p. 1071-1078.

9. Yan, C., et al., Evaluations of cellular lattice structures manufactured using selective laser melting. International Journal of Machine Tools and Manufacture, 2012. 62: p. 32-38.

10. Gorguluarslan, R.M., et al., Design and fabrication of periodic lattice-based cellular structures. Computer-Aided Design and Applications, 2016. 13(1): p. 50-62.

11. Kinstlinger, I.S., et al., Open-Source Selective Laser Sintering (OpenSLS) of Nylon and Biocompatible Polycaprolactone. PloS one, 2016. 11(2): p. e0147399.

12. Maskery, I., et al., An investigation into reinforced and functionally graded lattice structures. Journal of Cellular Plastics, 2016: p. $0021955 X 16639035$.

13. Gandhi, U., et al., DESIGNING LATTICE STRUCTURES FOR 3D PRINTING.

14. Ahmadi, S.M., et al., Mechanical behavior of regular open-cell porous biomaterials made of diamond lattice unit cells. J Mech Behav Biomed Mater, 2014. 34: p. 106-15.

15. Gibson, L.J. and M. Ashby. The mechanics of three-dimensional cellular materials. in Proceedings of the Royal Society of London A: Mathematical, Physical and Engineering Sciences. 1982. The Royal Society.

16. Ashby, M.F. and R.M. Medalist, The mechanical properties of cellular solids. Metallurgical Transactions A, 1983. 14(9): p. 1755-1769.

17. Onck, P., E. Andrews, and L. Gibson, Size effects in ductile cellular solids. Part I: modeling. International Journal of Mechanical Sciences, 2001. 43(3): p. 681-699.

18. Brenne, F., T. Niendorf, and H.J. Maier, Additively manufactured cellular structures: Impact of microstructure and local strains on the monotonic and cyclic behavior under 
uniaxial and bending load. Journal of Materials Processing Technology, 2013. 213(9): p. $1558-1564$.

19. Youssef, S., E. Maire, and R. Gaertner, Finite element modelling of the actual structure of cellular materials determined by X-ray tomography. Acta Materialia, 2005. 53(3): p. 719 730.

20. Smith, M., Z. Guan, and W.J. Cantwell, Finite element modelling of the compressive response of lattice structures manufactured using the selective laser melting technique. International Journal of Mechanical Sciences, 2013. 67: p. 28-41.

21. Park, S.-I., et al., Effective mechanical properties of lattice material fabricated by material extrusion additive manufacturing. Additive Manufacturing, 2014.

22. Jin, T., et al., Experimental study on the effects of specimen in-plane size on the mechanical behavior of aluminum hexagonal honeycombs. Materials Science and Engineering: A, 2015. 635: p. 23-35.

23. Zarringhalam, H., et al., Effects of processing on microstructure and properties of SLS Nylon 12. Materials Science and Engineering: A, 2006. 435-436: p. 172-180.

24. Lee, H.-H., Finite Element Simulations with ANSYS Workbench 14. 2012: SDC publications.

25. Hunt, H., The mechanical strength of ceramic honeycomb monoliths as determined by simple experiments: Advanced materials. Chemical engineering research \& design, 1993. 71(3): p. 257-266. 